\title{
Motor Pattern Specification by Dual Descending Pathways to a Lobster Rhythm-Generating Network
}

\author{
Denis Combes, Pierre Meyrand, and John Simmers \\ Laboratoire de Neurobiologie des Réseaux, Université Bordeaux I and Centre National de la Recherche Scientifique, Unité \\ Mixte de Recherche 5816, 33405 Talence, France
}

In the European lobster Homarus gammarus, rhythmic masticatory movements of the three foregut gastric mill teeth are generated by antagonistic sets of striated muscles that are driven by a neural network in the stomatogastric ganglion. In vitro, this circuit can spontaneously generate a single (type I) motor program, unlike in vivo in which gastric mill patterns with different phase relationships are found. By using paired intrasomatic recordings, all elements of the gastric mill network, which consists mainly of motoneurons, have been identified and their synaptic relationships established. The gastric mill circuit of Homarus is similar to that of other decapod crustaceans, although some differences in neuron number and synaptic connectivity were found. Moreover, specific members of the lobster network receive input from two identified interneurons, one excitatory and one inhibitory, that project from each rostral commissural ganglion. Integration of input from these projection elements is mediated by synaptic interactions within the gastric mill network itself. In arrhythmic preparations, direct phasic stimulation of the previously identified commissural gastric (CG) interneuron evokes gastric mill output similar to the type I pattern spontaneously expressed in vitro and in vivo. The newly identified gastric inhibitor interneuron makes inhibitory synapses onto a different subset of gastric mill neurons and, when activated with the CG neuron, drives gastric mill output similar to the type II pattern that is only observed in the intact animal. Thus, two distinct phenotypes of gastric mill network activity can be specified by the concerted actions of parallel input pathways and synaptic connectivity within a target central pattern generator.

Key words: Crustacea; Homarus gammarus; stomatogastric nervous system; central pattern generator; projection interneurons; network reconfiguration
It is now well established that most rhythmic motor behaviors are generated by neural networks, called central pattern generators (CPGs), that can operate in the absence of feedback from peripheral sense organs (Delcomyn, 1980). Nevertheless, to be functionally meaningful in vivo, CPGs must be susceptible to sensory and/or central modulatory input to adapt their motor output to changing behavioral demands. Thus, a given motor network must be able to alter its expression on a cycle by cycle basis, as well as over prolonged periods of ongoing activity. Several mechanisms by which a CPG can undergo functional reorganization have been proposed (Getting and Dekin, 1985). Two of these rely on modifications within the network itself, involving neuromodulation of synaptic interactions among constituent neurons and/or alterations in their intrinsic membrane properties (Marder and Calabrese, 1996). A third mechanism involves changes in the way sensory inputs impinge on the network, although a full understanding of this mechanism is primarily lacking because of the complexity and diversity of the afferent pathways involved (Soffe, 1997).

A preparation well suited to address this problem is the gastric mill system controlling rhythmic masticatory movements in the foregut of decapod crustaceans. Gastric mill movements are driven by the gastric mill motor network of the stomatogastric nervous system (STNS). This network can remain spontaneously

\footnotetext{
Received Oct. 10, 1998; revised Feb. 9, 1999; accepted Feb. 12, 1999.

This work was supported in part by the Human Frontier Science Program.

Correspondence should be addressed to Denis Combes, Laboratoire de Neurobiologie des Réseaux, Université Bordeaux I and Centre National de la Recherche Scientifique, Unité Mixte de Recherche 5816, Avenue des Facultés, 33405 Talence, France.

Copyright (ㄷ) 1999 Society for Neuroscience $\quad 0270-6474 / 99 / 193610-10 \$ 05.00 / 0$
}

active in vitro, and in several species the cellular properties and synaptic connectivity of its constituent neurons are known in detail (Selverston and Moulins, 1987; Harris-Warrick et al., 1992). Although a number of modulatory input pathways and their long-lasting influences on the gastric mill CPG have been elucidated (Meyrand et al., 1994; Norris et al., 1994; Blitz and Nusbaum, 1997), the role of "conventional" synaptic inputs, such as those arising from movement-related sensory feedback, in mediating rapid short-term reorganization of gastric mill motor output is still poorly understood.

In the present in vitro study, we describe the synaptic wiring of the previously incompletely known gastric mill CPG of the European lobster Homarus gammarus and analyze the influence of two identified interneurons that project from more rostral ganglia. One of these projection interneurons, the commissural gastric (CG) neuron (Robertson and Moulins, 1981; Simmers and Moulins, 1988) directly excites several gastric mill CPG elements. A second projection interneuron, the gastric inhibitor (GI) neuron, is identified in this study for the first time and exerts conventional inhibitory effects on several different gastric mill neurons. We show here that, in the absence of spontaneous rhythmicity, activation of these two input pathways, either separately or in combination, elicits distinct patterns of gastric mill network activity that resemble different rhythmic patterns observed in vivo. Importantly, the integration of these extrinsic influences strongly depends on indirect network-mediated interactions within the gastric mill circuit itself.

Parts of this work have been published in abstract form (Combes et al., 1995). 

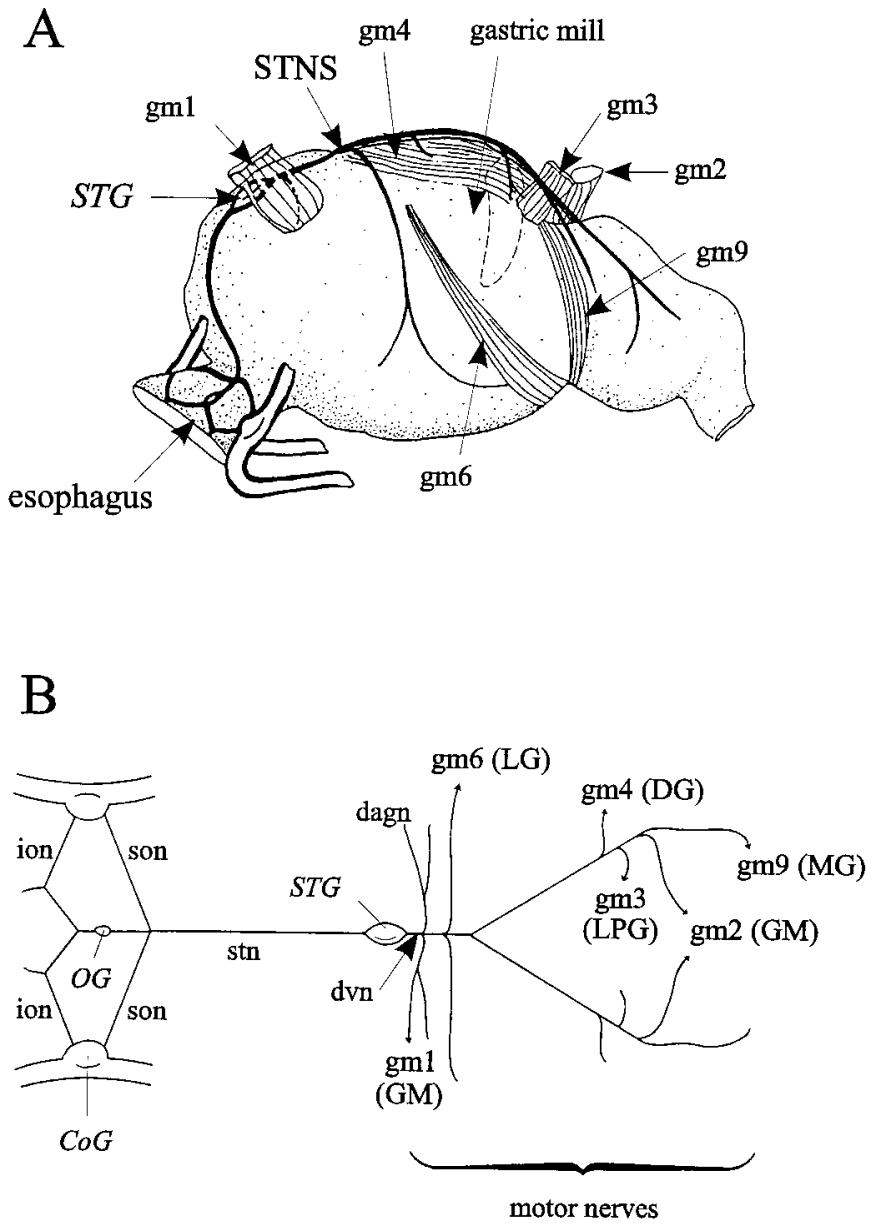

Figure 1. STNS of the lobster Homarus gammarus. A, Left lateral view of the foregut showing the STNS in situ and gastric mill muscles gm1-4, 6, and 9. B, STNS in vitro. The somata of gastric mill motoneurons (GM, LG, MG, DG, and LPG) are located in the STG, and their axons project caudally via the dorsal ventricular nerve $(d v n)$ into their respective bilateral motor nerve roots. The two CoGs and the OG are connected to the STG via the bilateral inferior oesophageal nerves (ions), the superior oesophageal nerves (sons), and the single, mainly afferent stn.

\section{MATERIALS AND METHODS}

All experiments were performed on adult European rock lobsters, Homarus gammarus, purchased from local commercial suppliers and kept in aquaria with fresh running seawater maintained at $16^{\circ} \mathrm{C}$. Before experiments, animals were cold anesthetized in ice for $\sim 30 \mathrm{~min}$. For in vitro experiments, the stomatogastric nervous system (STNS) was isolated from the foregut (Fig. 1A) as described by Combes et al. (1993) and consisted of the stomatogastric ganglion (STG), bilateral commissural ganglia $(\mathrm{CoG})$, the single oesophageal ganglion $(\mathrm{OG})$, and their interconnecting nerves, including the single stomatogastric nerve (stn) (Fig. $1 B$ ). Single motor axon types were obtained by dissecting out distal gastric mill motor nerve branches to their corresponding muscles (Fig. $1 A$ ). The STNS was then transferred to a Petri dish and superfused continuously with aerated artificial seawater maintained at $15-18^{\circ} \mathrm{C}$ with a thermoelectric cooling system (Midland Ross Inc.). The saline composition was (in mM): $\mathrm{NaCl} 479.12, \mathrm{KCl} 12.74, \mathrm{CaCl}_{2}-2 \mathrm{H}_{2} \mathrm{O} 13.67, \mathrm{MgSO}_{4}$ 10, $\mathrm{Na}_{2} \mathrm{SO}_{4}$ 3.91, and HEPES 5, buffered to $\mathrm{pH} 7.45$.

Extracellular recordings were made with platinum wire electrodes placed against appropriate nerves and isolated electrically with Vaseline. Intracellular recordings were made with glass microelectrodes placed in the soma or the neuropile of neurons after desheathing the corresponding ganglion. Microelectrodes (tip resistance of 10-30 M $\Omega$ ) were filled with $3 \mathrm{M} \mathrm{KCl}$ for recording-stimulation or with $2.5 \mathrm{M} \mathrm{LiCl}$ and $3 \%$ Lucifer yellow (Sigma, Quentin Fallavier, France) for labeling neurons. Dye was injected iontophoretically with negative current (5-10 nA, 20 min) and visualized with blue light illumination (450-490 nm). In some experiments, intense illumination for $30 \mathrm{~min}$ or more was used to photoinactivate injected neurons (Miller and Selverston, 1979).

Motoneurons were identified by correlating intrasomatic spikes with action potentials recorded along terminal motor nerves branches (see above). After impalement, the CG and GI projection neurons were identified in each $\mathrm{CoG}$ by their axonal projections in the superior oesophageal nerve (son) and the stn, and according to their different postsynaptic effects on gastric mill motoneurons.

In several experiments, electromyographic recordings were made from gastric mill muscles of freely behaving lobsters using EMG recording methods as described previously (Clemens et al., 1998). Briefly, Tefloninsulated silver wire electrodes (diameter of $125 \mu \mathrm{m}$ ) were implanted into appropriate muscles via holes drilled in the cephalothorax and connected via flexible wires to laboratory-constructed extracellular amplifiers. Conventional techniques for in vivo and in vitro experiments were used for display, storage, and transcription of recorded data.

Labeling of the gastric motoneuron (GM) population was also made by passive axonal migration of cobalt chloride ( $8.5 \%$ in distilled water) from the cut end of the dorsal anterior gastric nerve (dagn) (Fig. 1B) toward the STG. Migration occurred for $36 \mathrm{hr}$ at $4^{\circ} \mathrm{C}$, and then the preparation was treated with $2 \%$ ammonium sulfide, fixed for $1 \mathrm{hr}$ in $2.5 \%$ glutaraldehyde, dehydrated, and cleared in methyl salicilate (Simmers and Moulins, 1988).

\section{RESULTS}

\section{The gastric mill system of Homarus}

Muscles responsible for rhythmic movements of the one medial and two lateral gastric mill teeth in the lobster foregut and their innervation by the STNS are illustrated in Figure $1 A$ (Maynard and Dando, 1974; Hartline and Maynard, 1975; Selverston and Moulins, 1987). The medial tooth produces alternate phases of protraction and retraction mediated by the gm 1 and gm 2 muscles (innervated by GMs) and the gm4 muscle [innervated by the dorsal gastric (DG) motoneuron], respectively. Alternate closing and opening movements of the lateral teeth are driven by the gm6 and gm9 [innervated by lateral gastric (LG)-medial gastric (MG) motoneurons] and gm3 [innervated by lateral posterior gastric (LPG) motoneurons] muscles, respectively (Fig. 1 $A, B$ ).

\section{Spontaneous gastric mill output patterns}

Typical spontaneous rhythmicity of the gastric mill network in vitro is illustrated in Figure $2 A$. This version of gastric mill motor output (which we designate "type I") consists of MG and GM motoneurons, which innervate lateral and medial teeth powerstroke muscles, firing synchronous bursts in antiphase with motoneurons (Fig. 2A, LPG trace) that innervate antagonistic return-stroke muscles.

As seen in the EMG recordings from gastric mill muscles in Figure $2 B_{1}$ (S. Clemens, personal communication), the type I pattern is also expressed in vivo ( $\sim 80 \%$ of recorded animals). Again, the lateral teeth opener muscle gm3 (innervated by the LPG neurons) is activated in antiphase with the medial tooth protractor muscle gm2 (innervated by the GM motoneurons). However, unlike in vitro, in the same intact lobster, other types of gastric mill network activity are also observed (Heinzel, 1988). One of these different versions, which we refer to as "type II," is illustrated in Figure $2 B_{2}$. In this sequence, which is spontaneously expressed $<10 \%$ of the time, medial tooth protraction and lateral teeth opening (driven by GM and LPG motoneuron-innervated muscles, respectively) now occur conjointly (Figure $2 \mathrm{~B}_{2}$, ellipse) and in antiphase with lateral teeth closing (driven by the MG motoneuron). Therefore, in vivo, a reorganization of gastric mill motor output can occur in which lateral and medial teeth movements become coordinated differently. 
A

in vitro

MG

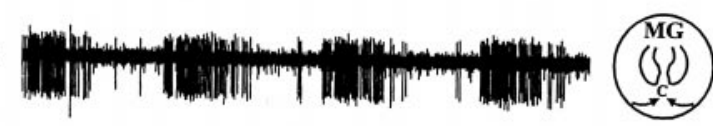

LPG

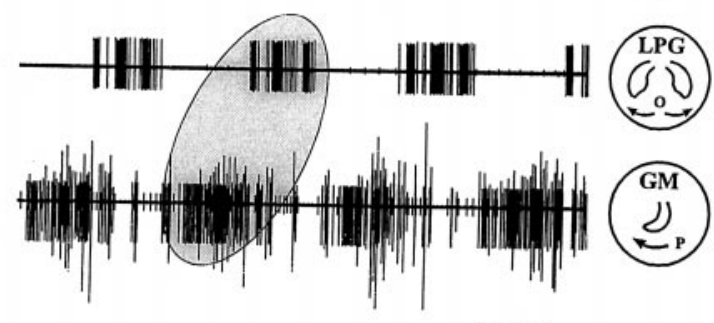

$2 \mathrm{~s}$

$\mathbf{B}_{1}$

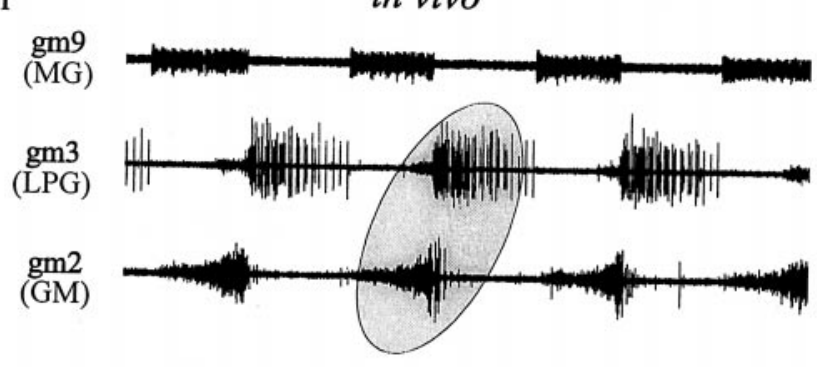

$\mathbf{B}_{2}$

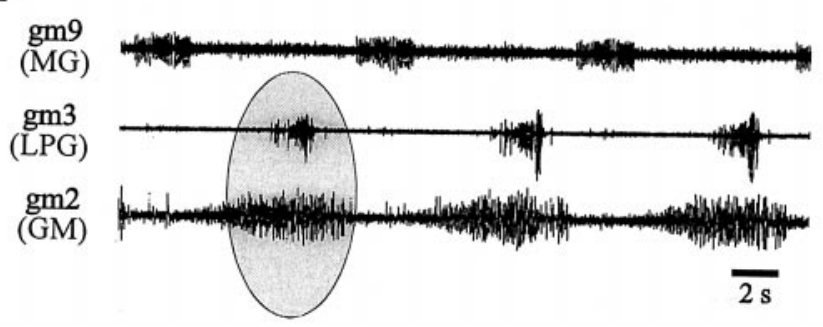

Figure 2. Gastric mill output patterns observed in an isolated STNS $(A)$ and in vivo $(B) . A$, Extracellular recordings of gastric mill motor nerves in vitro. GM motoneurons (medial tooth protraction) fire in antiphase with LPG motoneurons (lateral teeth opening; ellipse) and in phase with the MG motoneuron (lateral teeth closure). Icons on the right show teeth movements produced by each motoneuron subgroup ( $P$, protraction; $O$, opening; $C$, closing). $B$, Electromyographic recordings of gastric mill muscles in vivo showing two distinct coordination patterns. $B_{1}$, Pattern similar to that seen in vitro (compare with $A$ ) in which muscles innervated by the LPG and GM motoneurons are alternately active (ellipse). $B_{2}$, Different animal in which the muscles innervated by the LPG and GM motoneurons are coordinately active (ellipse). In this pattern, protraction of the medial tooth (GM neurons) occurs with lateral teeth opening (LPG neurons).

\section{The gastric mill network of $\mathrm{H}$. gammarus}

In a first step toward exploring the neural basis of these different gastric mill rhythms, we established the hitherto undescribed synaptic connectivity of the gastric mill motor network in $H$. gammarus. As seen in the pairwise intrasomatic recordings of Figure $3 A$, like in other crustaceans (Mulloney and Selverston, 1974a,b; Selverston and Moulins, 1987), inhibitory chemical synapses predominate in the $H$. gammarus gastric mill network. For example, the LG neuron inhibits the LPG neurons, the DG neuron and interneuron 1 (Int1), and in each case the short latency and 1:1 relationship between IPSP and presynaptic impulse suggests a monosynaptic relationship. (Note that for the two electrically coupled LPG neurons, because we did not perform
A
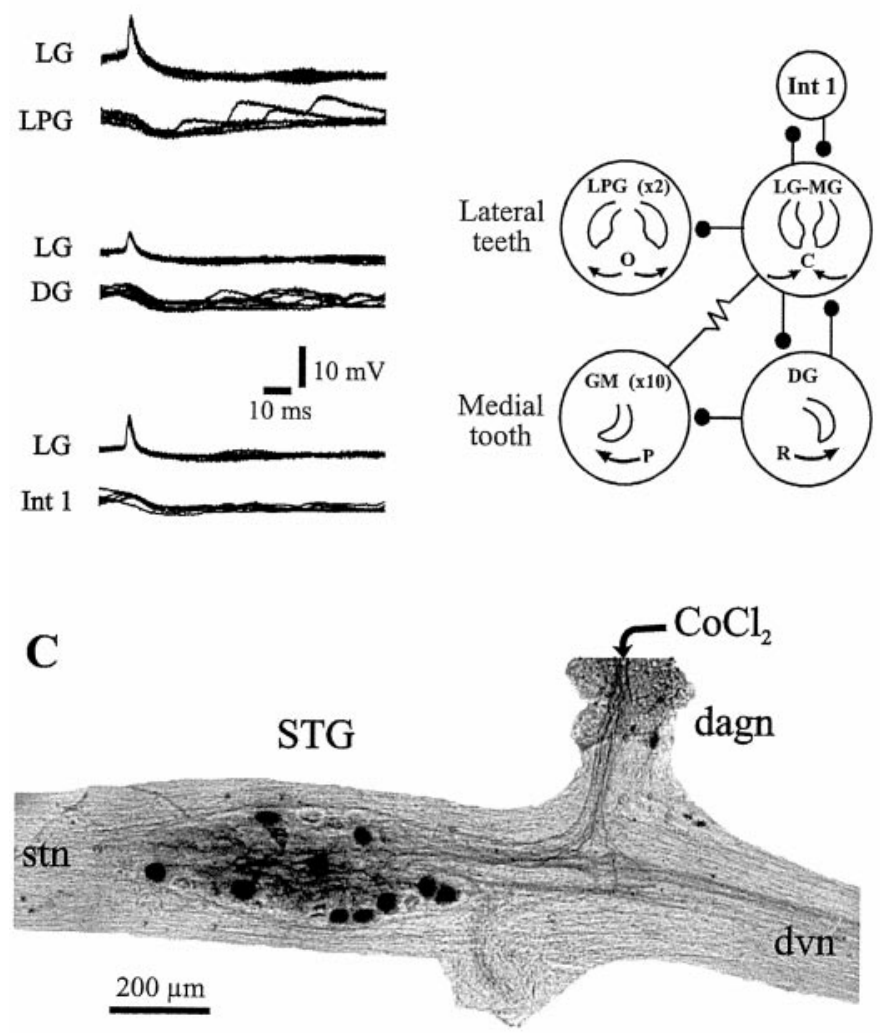

Figure 3. Gastric mill network of $H$. gammarus. A, Dual intrasomatic recordings that reveal probable monosynaptic inhibitory connections. Each panel shows superimposed oscilloscope sweeps triggered by impulses in the presynaptic LG neuron. $B$, Synaptic wiring diagram derived from recordings as in $A$. Stick and ball symbol, Chemical inhibitory synapse; resistor symbol, electrical coupling. Numbers denote the number of neurons of each type. Neurons of each functional group are electrically coupled, including the LG-MG neurons. Tooth movement driven by each neuron type is also indicated: $O, C$, opening and closing of lateral teeth, respectively; $P, R$, protraction and retraction of medial tooth, respectively. $C$, Anatomical evidence for $10 \mathrm{GM}$ motoneurons after cobalt $\left(\mathrm{CoCl}_{2}\right)$ backfill from their axons in the dagn; 10 STG somata are labeled.

cell kills, we cannot tell whether one or both cells actually receive direct LG neuron-mediated IPSPs). As in other Crustacea (Selverston and Moulins, 1987), the single LG and MG motoneurons are strongly electrically coupled and appear to behave as a functional entity. Strong electrical coupling also exists between the GM and LG-MG motoneurons (Fig. 3B; also see below). The 15 motoneurons [in addition to the solitary interneuron (Int1)] that constitute the lobster gastric mill network are classified into four subgroups; two of these subsets innervate the opener (two LPG motoneurons) and closer muscles (LG and MG motoneurons) of the lateral teeth subsystem, whereas the remaining two subgroups innervate the protractor (GM motoneurons) and retractor (one DG motoneuron) muscles of the medial tooth subsystem. Neurons within each functional group (LG-MG, GM, and LPG neurons) are also electrically coupled.

Unlike spiny lobsters and crabs, which possess only four GM motoneurons (Mulloney and Selverston, 1974a,b; Selverston and Moulins, 1987), H. gammarus has $10 \mathrm{GM}$ neurons. This is demonstrated in Figure $3 C$, which shows a cobalt backfill of the dagn, which carries all of the GM neuron axons. In 4 of 4 preparations, 10 STG somata were labeled. This anatomical finding was con- 

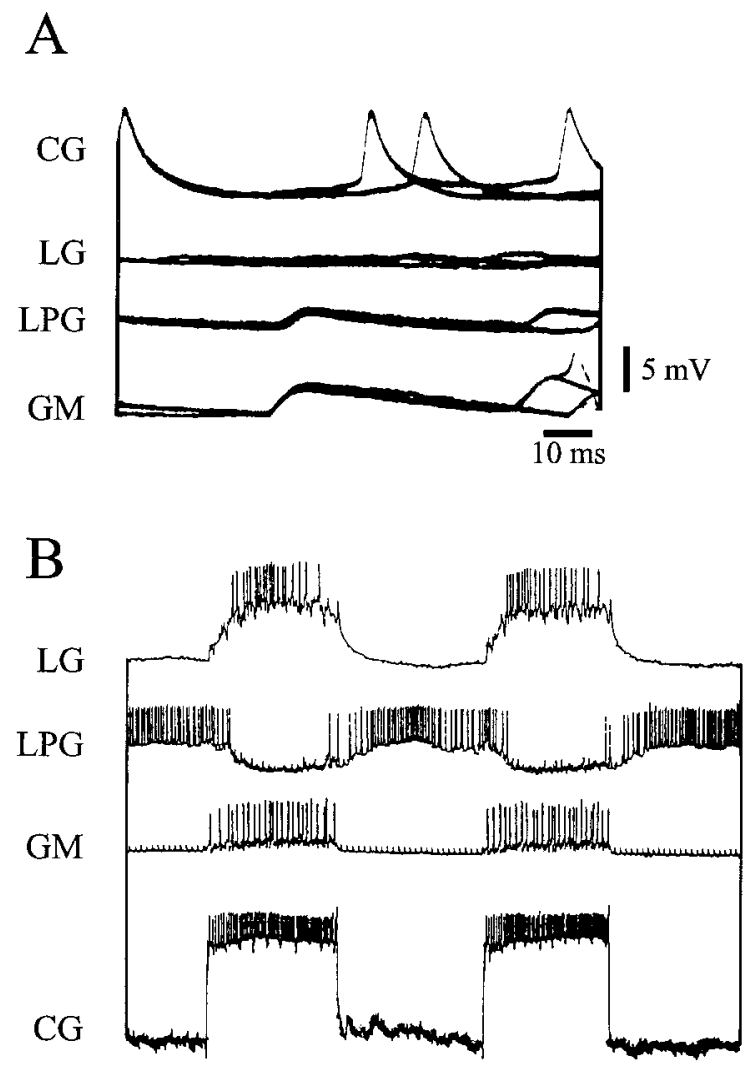
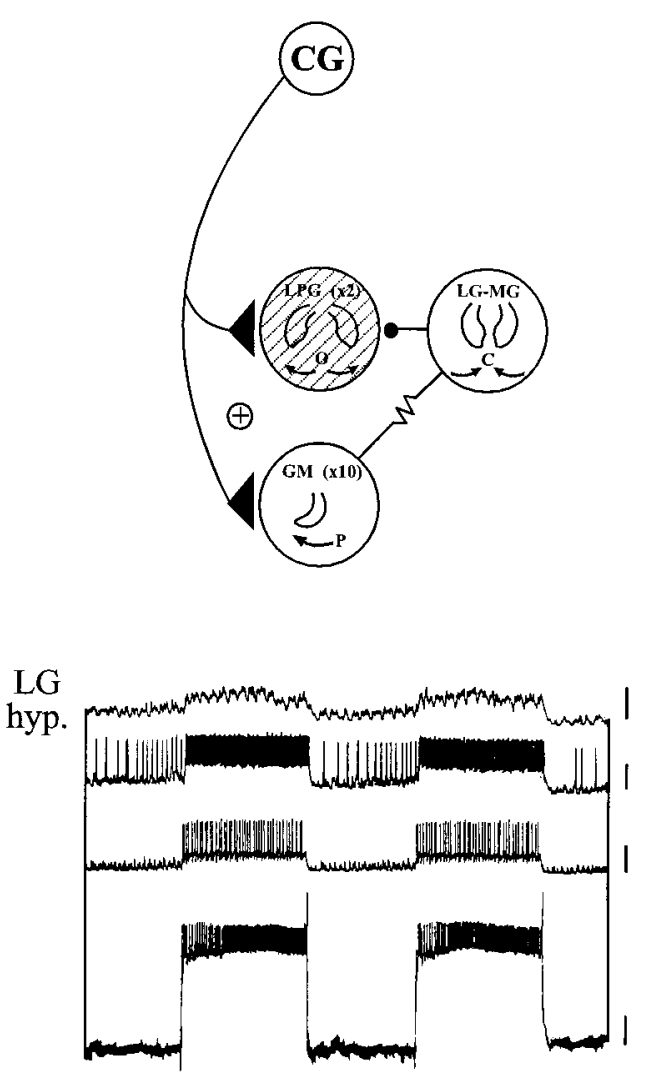

Figure 4. CG interneuron interactions with the gastric mill network. $A$, Superimposed sweeps showing CG neuron-derived EPSPs in the LPG and GM neurons but not in the LG neuron. Schema at top right shows the synaptic relationship between the CG neuron and the gastric mill network. $B$, Suprathreshold depolarization of the CG neuron excites the GM and LG neurons but inhibits the LPG neuron (left). However, when the LG neuron is continuously hyperpolarized by intracellular current injection (right), CG neuron stimulation now causes coordinated LPG and GM neuron excitation. Calibration: vertical bars, $10 \mathrm{mV}$; horizontal bar, 2 sec.

firmed by systematic intracellular recording from all STG neurons; in 3 of 3 preparations, 8-10 GM neurons could be identified electrophysiologically.

\section{Gastric mill CPG flexibility: role of projection interneurons and network connectivity}

To understand how the gastric mill network can express the distinctly different motor programs seen in vivo (Fig. $2 B$ ), we investigated inputs to the network that might be responsible. More precisely, because in the type II pattern (Fig. $2 \mathrm{~B}_{2}$ ), the LPG and GM motoneurons fire together instead of in alternation as in the type I pattern (Fig. $2 B_{1}$ ), we sought an extrinsic excitatory influence that could force these two subgroups to fire in phase rather than in antiphase.

\section{CG interneuron}

A source of excitatory input to the gastric mill network is the pair of CG interneurons. First identified by Robertson and Moulins (1981), the single CG neuron in each CoG projects its axon to the STG via the ipsilateral son and the stn (Fig. 1). It was also shown previously that in Homarus (Simmers and Moulins, 1988) each CG neuron makes direct excitatory synapses onto the GM motoneurons. We show here that the $\mathrm{CG}$ neurons also directly excite the LPG neurons. As seen in Figure 4A, each CG neuron spike is always followed at constant delay by EPSPs in the GM and LPG neurons. The monosynapticity of these synaptic responses is further suggested by their persistence in high $\mathrm{Ca}^{2+}-\mathrm{Mg}^{2+}$ saline (data not shown) (Simmers and Moulins, 1988). In contrast, no discrete synaptic activity arising from the $\mathrm{CG}$ neuron was observed in LG-MG neuron recordings (Fig. 4A).

To test whether the CG neuron could be responsible for the GM-LPG neuron coactivation seen in the type II gastric mill
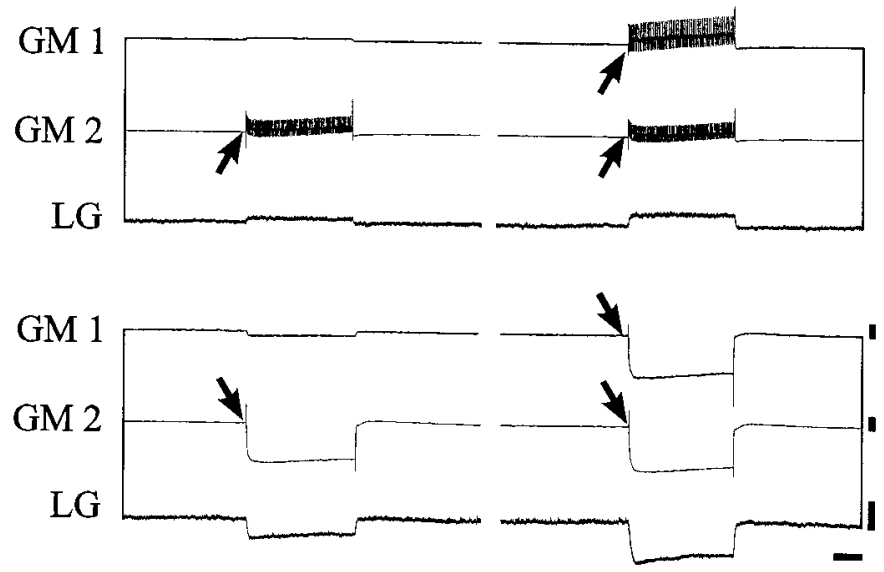

Figure 5. Electrical coupling between GM and LG motoneurons. Depolarization of one (top left) or two (top right) GM neurons by current injection (arrows) depolarizes the LG neuron. Hyperpolarization of one (bottom left) or two (bottom right) GM neurons by current injection (arrows) hyperpolarizes the LG neuron. Note the increased postsynaptic response when both GM neurons are manipulated with current. Calibration: vertical bars, $20 \mathrm{mV}$; horizontal bar, $1 \mathrm{sec}$. 

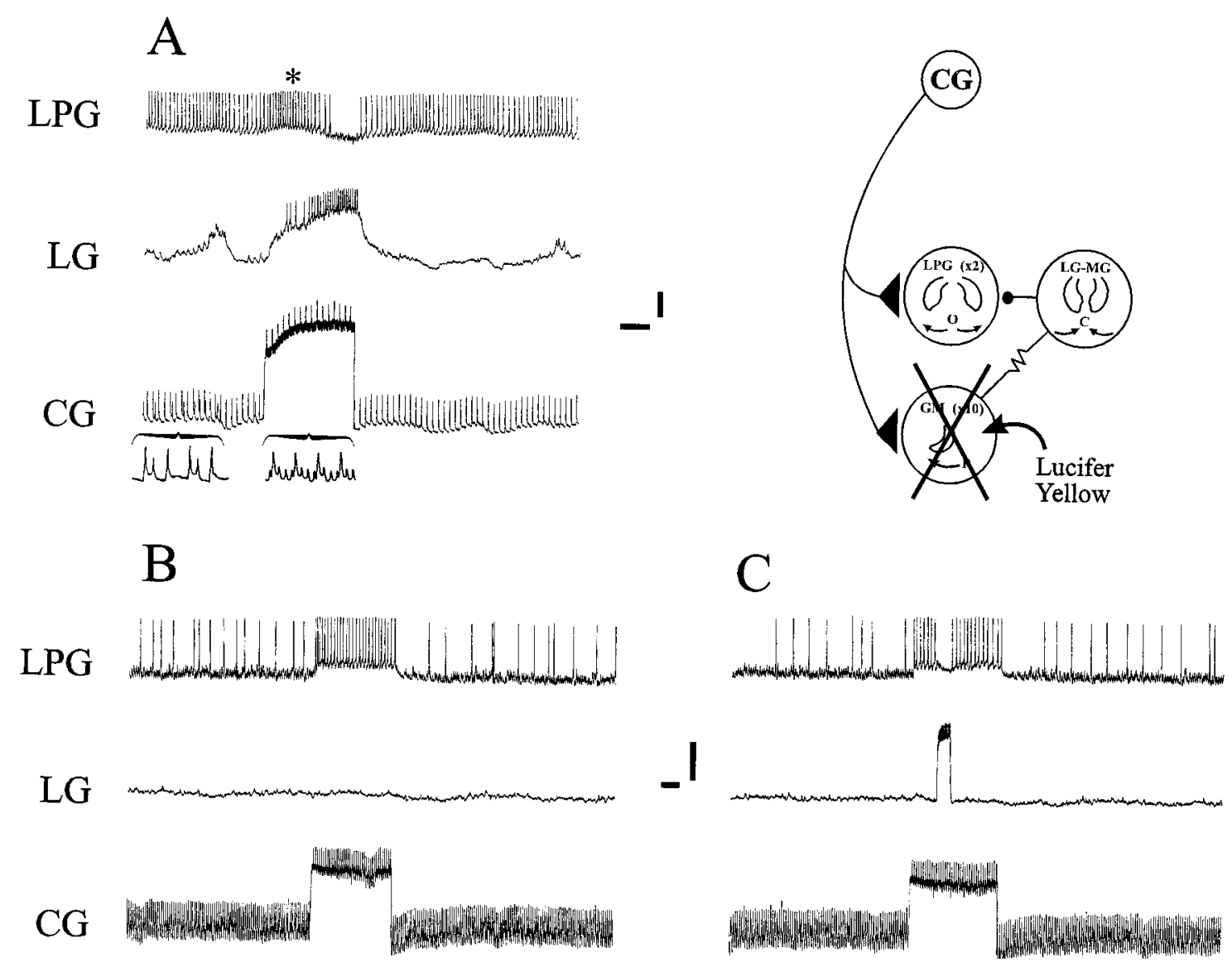

Figure 6. The CG interneuron excites the LG neuron via the GM neurons. A, Stimulation of the CG neuron by intracellular current injection excites the LG neuron and, after a slight excitation (asterisk), inhibits an LPG neuron. Note that the smaller depolarizing transients in the CG neuron are action potentials, whereas the larger potentials are EPSPs (insets are CG neuron recordings on faster time base). B, After photoinactivation of Lucifer yellow-filled GM neurons by intense blue light illumination, CG neuron depolarization no longer activates the LG neuron and now excites the LPG neurons. $C$, LG neuron activation by current injection during CG neuron stimulation still causes LPG neuron inhibition. Calibration: vertical bars, 10 $\mathrm{mV}$; horizontal bars, $1 \mathrm{sec}$.

pattern, the interneuron was periodically stimulated with pulses of depolarizing current while recording from neurons of the different gastric mill network subgroups. As seen in Figure 4B, left, cyclic CG neuron firing activated the GM motoneuron (third trace) but surprisingly inhibited the LPG neurons (second trace). In contrast, the $\mathrm{LG}$ neuron, which receives no direct input from the CG neuron (Fig. 4A), was also strongly excited during the activity of the interneuron. The explanation for this apparent paradox lies in the synaptic interactions within the gastric mill network itself. Specifically, the LG-MG neuron inhibition of the LPG neuron overcomes the CG neuron excitation. This opposing synaptic effect is revealed in Figure $4 B$, right, in which the LG neuron was held continuously hyperpolarized and silent during CG neuron stimulation. With the LG neuron (and the electrically coupled MG neuron) thus functionally removed from the network, each burst evoked in the CG interneuron now elicited synchronous spike trains in the LPG and GM motoneurons (Fig. $4 B$, compare right, left panels). Therefore, hyperpolarization of the LG neuron released the LPG neuron from the LG neuron inhibition and permitted it to fire in response to direct excitation from the CG neuron.

Given that the LG-MG neuron group does not receive synaptic input from the CG neuron, how is the LG-MG neuron group activated, and hence the LPG neurons inhibited, by CG neuron activity? Here, again, the most likely explanation is a networkmediated effect in which LG neuron activation during CG neuron firing is caused by the strong GM-LG neuron electrical coupling (Fig. 4, schema). Several experimental arguments support this conclusion. First, excitation by the CG neuron of all 10 GM motoneurons provides a powerful drive to the LG-MG neurons. This combined electrotonic action of the GM neuron ensemble can be seen in Figure 5 in which an LG neuron was recorded during current injection into one or two simultaneously recorded GM neurons. Both depolarization (top) and hyperpolarization (bottom) of the GM neurons caused a corresponding depolarizing or hyperpolarizing LG neuron response that was proportional to the number of manipulated GM neurons (Fig. 5, compare left, right side of each panel).

A second direct argument that inhibition of the LPG neurons by the gastric mill network (via the LG neuron) is stronger than the $\mathrm{CG}$ neuron excitation derives from experiments $(n=4)$ in which the GM neurons were removed from the gastric mill network by photoinactivation (Fig. 6). In the preparation illustrated in Figure $6 A$, the gastric mill network was spontaneously arrhythmic under control conditions, and CG neuron depolarization caused excitation and firing in an LG neuron and inhibition of a tonically active LPG neuron (compare with Fig. 4B, left). Moreover, consistent with the mixed direct and indirect effects of the 
interneuron on the LPG neuron, the latter was initially excited (by direct CG neuron input; Fig. 6A, asterisk) when LG neuron firing was still weak. [Note that the larger depolarizing transients in the interneuron are EPSPs (Fig. 6A, bottom insets), a feature of the CG interneuron (Robertson and Moulins, 1981; Simmers and Moulins, 1988). The smaller depolarizing events are electrotonically decremented action potentials.] The entire population of GM neurons was then backfilled into the ganglion with Lucifer yellow placed in a Vaseline well built around their cut axons in the dagn (Fig. 1B). After $12 \mathrm{hr}$ of dye migration, the preparation was illuminated with blue light causing the photo-ablation of the Lucifer-stained motoneurons (schema). In this experimental situation, the CG neuron no longer excited the LG neuron (because the GM neurons were dead), and hence CG neuron activity was now able to directly excite the LPG neurons (Fig. 6B). That the silent LG neuron was still able to influence the LPG neuron is shown in Figure $6 C$ in which a brief LG depolarization caused a transient LPG neuron inhibition during CG neuron-evoked excitation. This experiment therefore strongly argues that, although the LPG neuron receives direct excitation from the CG interneurons, this effect is normally masked by inhibition from the LG-MG neuron group, which is itself activated as a consequence of its electrical coupling to the $10 \mathrm{CG}$ neuron-activated GM neurons.

Thus, two major conclusions arise from these results. First, despite its direct excitatory connection with both the GM and LPG motoneurons, activation of the projection CG neuron alone is unable to produce the gastric mill pattern in which the LPG and GM neurons are coordinately active (Fig. $2 B_{2}$ ). Second, experiments with current injection (Fig. $4 B$ ) and photoablation (Fig. 6B,C) suggest that such a network configuration is feasible only if the LG-MG neurons are inhibited so that excitation of the LPG neuron by the CG neuron can be expressed. We present below evidence for a newly described projection neuron with precisely such properties.

\section{Commissural GI interneuron}

The essential properties of this newly identified commissural interneuron, which we have called the gastric inhibitor neuron because of its effects on the gastric mill network (see below), are shown in Figures 7 and 8. The soma of the GI neuron lies medially in each $\mathrm{CoG}$, and its dendritic neuropile projects rostrally into a region in which the $\mathrm{CG}$ interneuron is also located (Simmers and Moulins, 1988) (Fig. 7A), although we have found no evidence of a synaptic connection between the two interneurons (Fig. 9A,B). Moreover, as with the two CG interneurons, the bilaterally paired GI neurons do not appear to be coupled synaptically (data not shown). The GI neurons project to the STG via the ipsilateral son and the stn (Fig. $7 B, C$ ). Stimulating a GI neuron with intracellular current inhibits the LG, MG, and DG neurons (Fig. 8A), and superimposed oscilloscope sweeps (Fig. $8 B$ ) reveal that each GI neuron action potential induces a constant latency IPSP (arrows) in all three neurons. These presumed direct synaptic influences of the GI neuron, which were not found on other gastric mill network neurons, are summarized in Figure 8, schema.

\section{Activation of the $\mathbf{C G}$ and $\mathbf{G I}$ projection neurons can reproduce types I and II gastric mill output patterns}

Finally, to examine and compare the actions of the excitatory CG and inhibitory GI neurons on the gastric mill network, we stimulated them separately and together in otherwise spontaneously
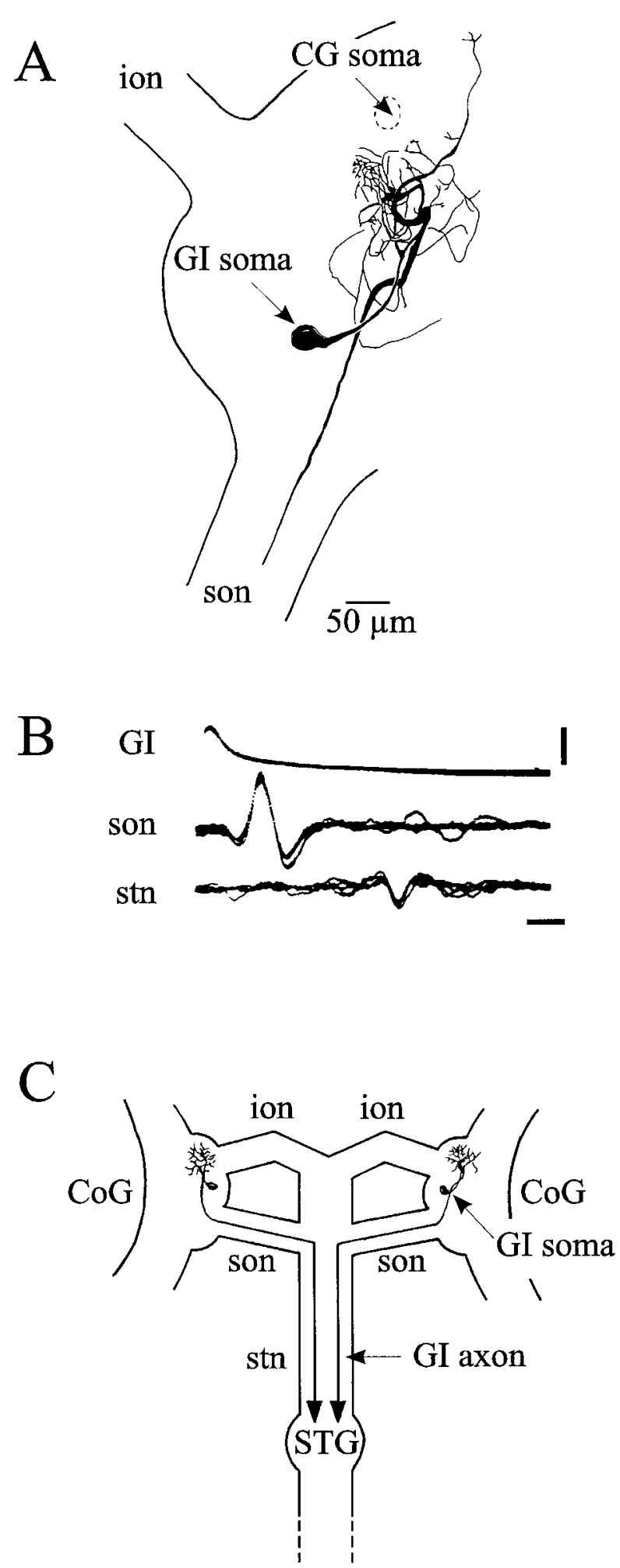

Figure 7. Identification of descending commissural GI interneuron. $A$, Camera lucida drawing of a Lucifer yellow-stained GI neuron in the right CoG; the GI neuron soma lies between the emergence of the ion and son through which its axon runs toward the stn. CG neuron soma position in the same $\mathrm{CoG}$ is also indicated. $B$, Each GI neuron spike is correlated 1:1 with an axonal spike recorded extracellularly in the son and then in the stn (5 superimposed oscilloscope sweeps). Calibration: vertical bar, $5 \mathrm{mV}$; horizontal bar, 2 msec. $C$, Geometry of GI neurons in the STNS; their somata are located one in each CoG and their axons project to the STG via the son and the stn. 

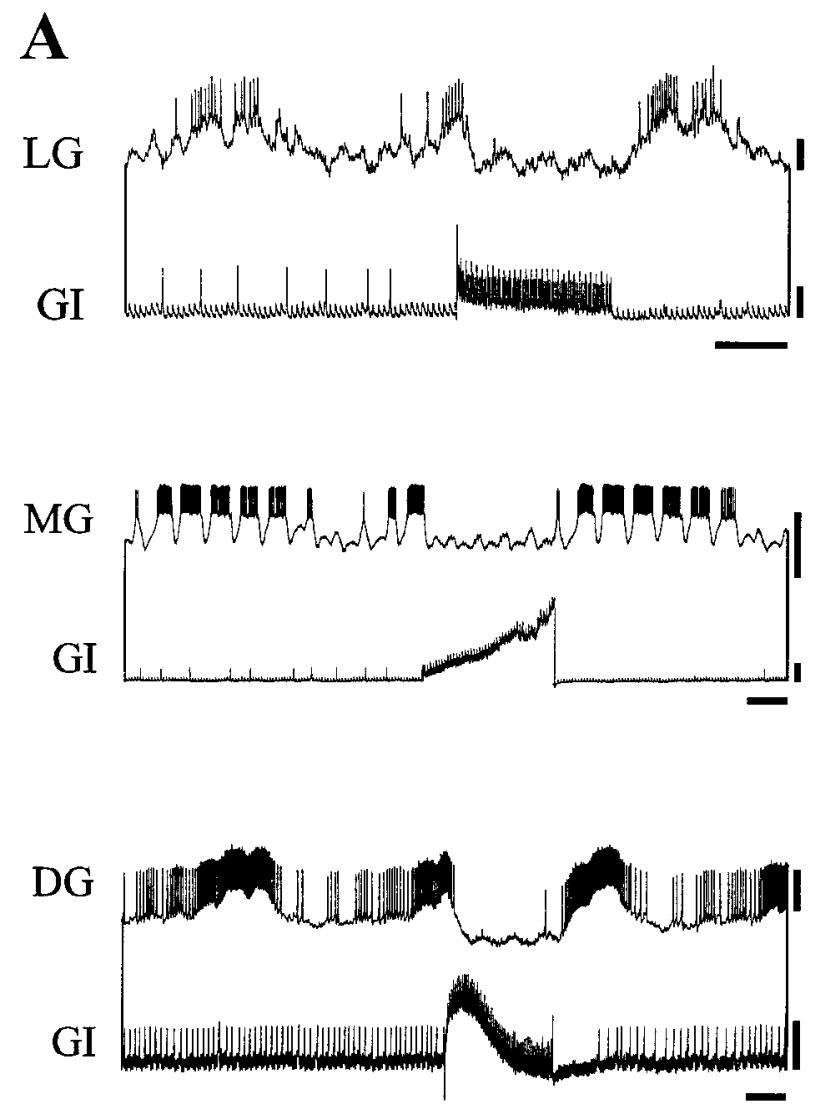

B
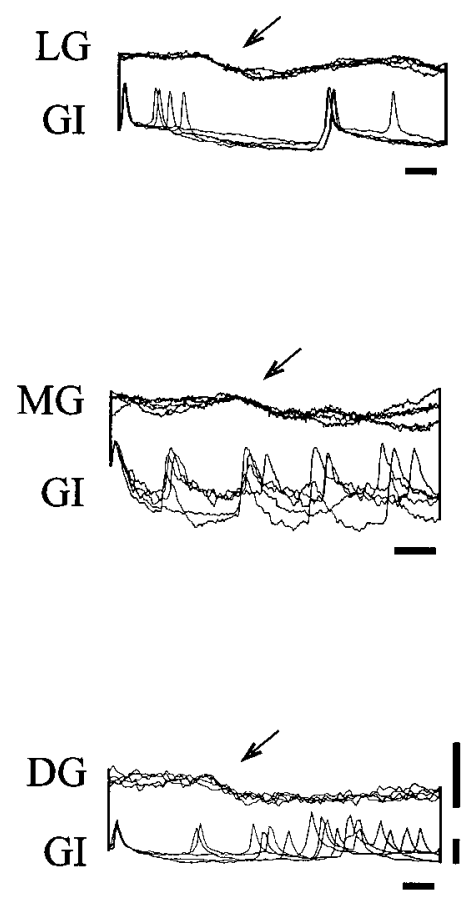

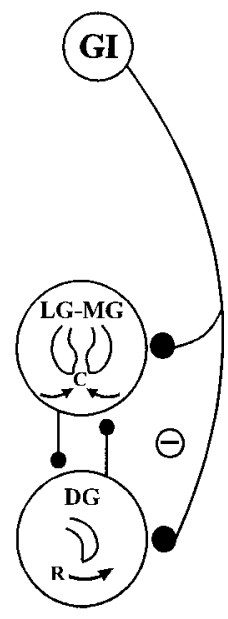

Figure 8. Synaptic relationship of the GI interneuron with the gastric mill network. $A$, Depolarization of the GI neuron inhibits the LG (top), MG (middle), and DG (bottom) neurons. B, Faster time base (5 sweeps in each recording). Each GI neuron spike is correlated 1:1 with a constant latency IPSP (arrows) in the MG, DG, and LG neurons. Diagram at right summarizes these synaptic relations. Calibration: vertical bars, $A, 10 \mathrm{mV} ; B, 5 \mathrm{mV}$; horizontal bars, $A, 2 \mathrm{sec} ; B, 10 \mathrm{msec}$.

arrhythmic preparations. To assess their capacity to elaborate coordinated fictive motor patterns similar to those generated spontaneously, the interneurons were driven with cyclic depolarizations at periods similar to those of gastric mill network rhythmicity. One of three such experiments in which both interneurons and the gastric CPG neurons were recorded simultaneously is illustrated in Figure 9, which also shows the combined synaptic wiring diagram of the descending interneurons and the motor network. First, rhythmic bursts evoked in the excitatory $\mathrm{CG}$ neuron alone (Fig. 9A) immediately elicited coordinated motor bursting similar to the type I gastric mill pattern that is normally spontaneously expressed under in vitro conditions (Fig. 2A). Thus, the MG and GM neuron bursts occurred in phase with CG neuron activity and in antiphase with the LPG neurons (ellipse). A pattern with a qualitatively similar phase relationship was also produced by rhythmic stimulation of the GI neuron alone (Fig. $9 B)$. Here, however, unlike the response to CG neuron activation, the MG and GM motoneurons fired in antiphase with the GI neuron. In contrast, coactivation of the two descending interneurons induced a gastric output similar to the type II pattern spontaneously expressed in vivo (Fig. $2 B$ ); the LPG and GM motoneurons now fired bursts conjointly and in time with the interneurons but in antiphase with the MG neuron (Fig. 9, compare $C$, ellipse, with $A, B$, ellipses). In all cases, the evoked pattern persisted for as long as the interneuron(s) was stimulated (data not shown). Therefore, by selective or simultaneous activation of two parallel projection pathways, replicas of distinct gastric mill motor patterns encountered in vivo can be generated in vitro, even in the absence of spontaneous network rhythmicity.

\section{DISCUSSION}

\section{Functional flexibility of the gastric mill network}

The purpose of this study was to explore the capability of a central motor pattern-generating network to produce different activity phenotypes seen in vivo. After determining the synaptic connectivity of the previously undescribed gastric mill CPG in the stomatogastric ganglion of Homarus gammarus, we show that appropriate motor pattern selection can occur as a result of extrinsic input from two types of projection neurons arising from each of the bilateral commissural ganglia. Thus, activation of the excitatory $\mathrm{CG}$ interneuron alone can drive a type I fictive gastric mill motor pattern, whereas simultaneous stimulation of both the GI and CG neurons immediately induces a type II-like gastric mill output pattern.

In the stomatogastric system of a number of decapod species, it is now established that different motor patterns can be elicited from the STG networks by activation of different projection neurons or the presence of neuromodulatory substances (Hooper and Marder, 1984; Harris-Warrick et al., 1992; Meyrand et al., 1994; Norris et al., 1994; Marder and Calabrese, 1996; Blitz and Nusbaum, 1997). In virtually all reported cases, however, the network reorganization appears to rely on long-lasting neuromodulatory instruction involving relatively slow changes in synaptic and cellular properties within the target CPG itself (Getting 


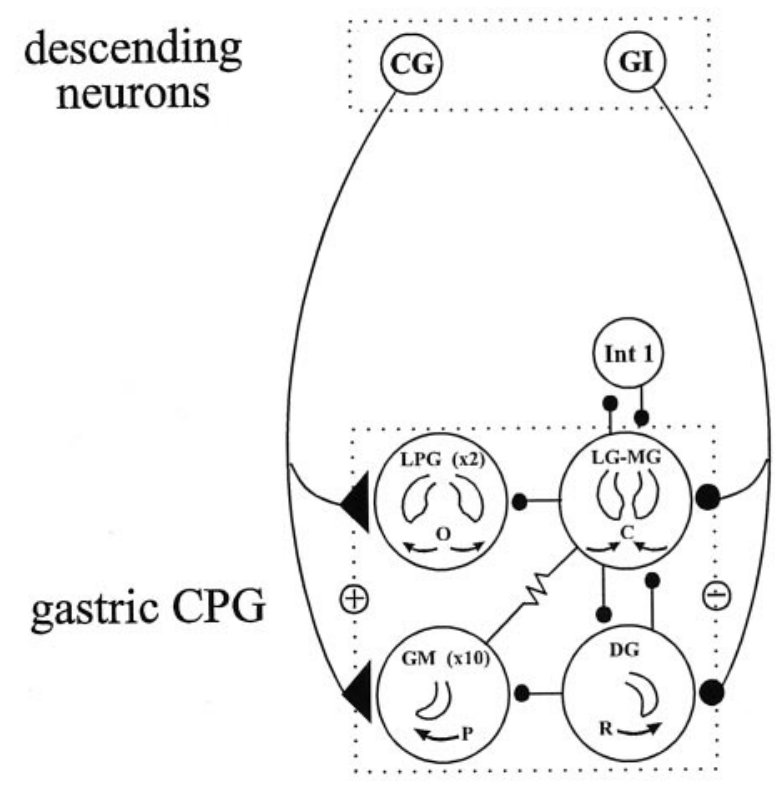

B

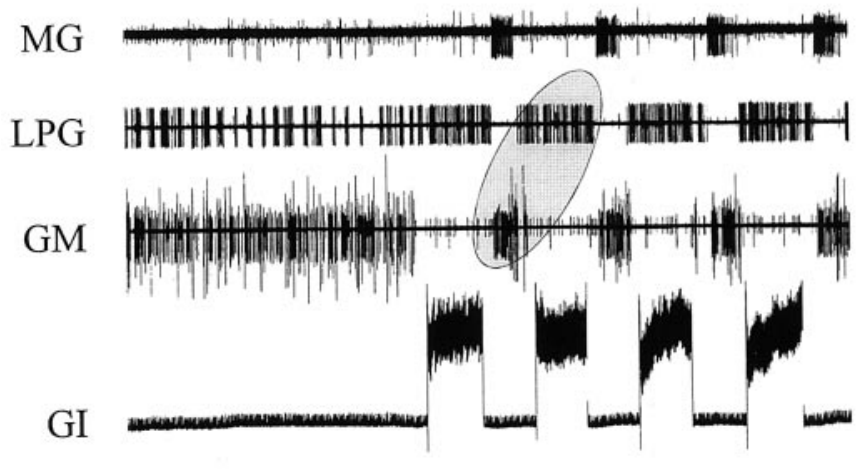

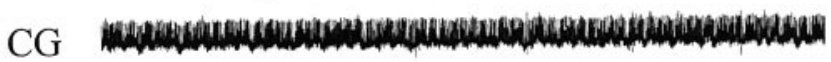

A $\mathrm{MG}$

LPG

GM
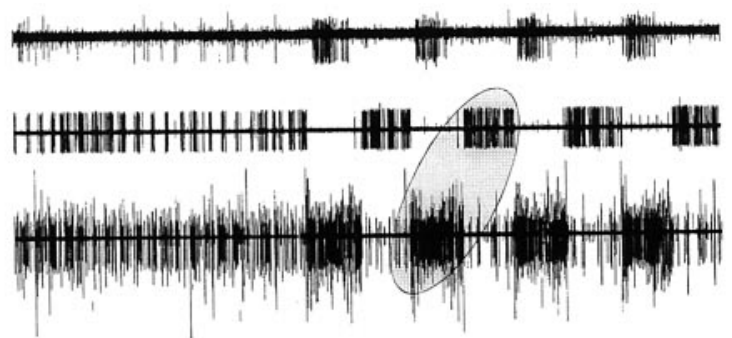

GI

CG

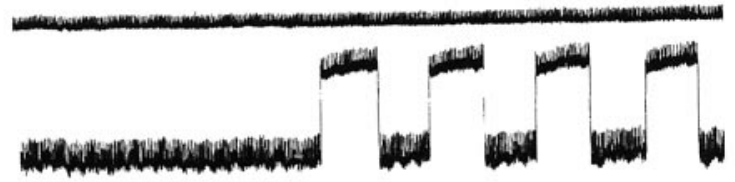

$10 \mathrm{mV}$

C

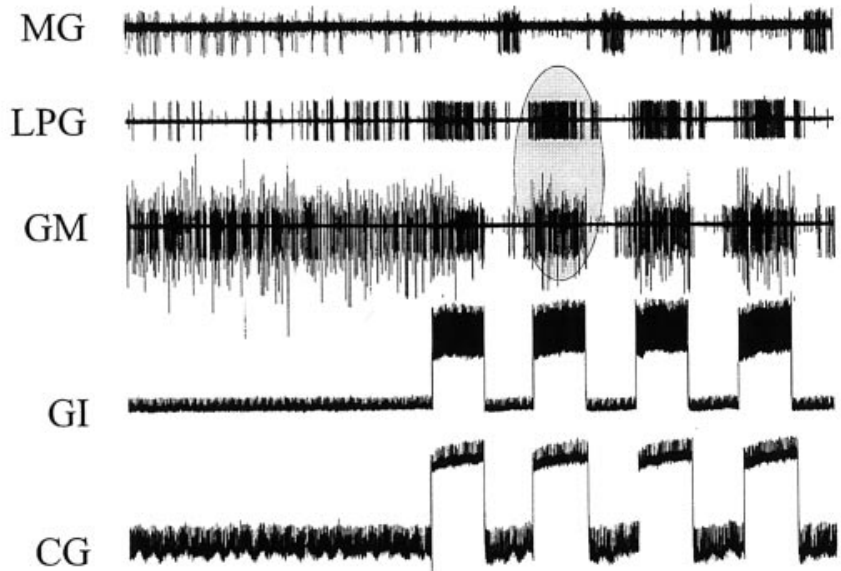

Figure 9. Different gastric mill patterns encountered in vivo can be reproduced in vitro by activating the CG and GI interneurons. Schema summarizes synaptic relationships between descending CG and GI neurons and the gastric mill network. $A$, Periodic depolarization of a tonically active CG neuron alone generates a gastric mill pattern that resembles a type I pattern (LPG and GM neurons fire out of phase; ellipse) seen spontaneously in vivo. B, In the same preparation, rhythmic activation of the GI neuron alone also produces a pattern in which LPG and GM neuron bursts are in phase opposition. Here, however, the LPG neuron fires in phase with the stimulated interneuron. $C$, Simultaneous activation of the CG and GI interneurons organizes gastric mill activity into a type II-like pattern, similar to that spontaneously expressed in vivo; here, the LPG and GM neurons fire in phase (ellipse) and in antiphase with the MG neuron.

and Dekin, 1985; Harris-Warrick et al., 1992; Morton and Chiel, 1994). To date, the ability of multiple input pathways, such as those mediating movement-related sensory feedback, to achieve rapid network reconfiguration is much less well documented. This is in large part attributable to the difficulty in identifying the ensemble of pathways involved, as well as a lack of knowledge of the way in which they impinge on their target network. The only other well characterized example of proprioceptive input in the stomatogastric system is an identified set of muscle receptors, the gastropyloric receptor (GPR) cells (Katz et al., 1989). Interestingly, the GPR cells exert classical fast-acting synaptic influences on several STG neurons, although here again, their primary action appears to be long-lasting neuromodulatory influences on intrinsic bursting properties (Katz and Harris-Warrick, 1989).

That the ongoing output of rhythmic neural networks can be modified by activation of previously silent input neurons has been reported. For example, in the locomotor systems of both the mollusc Clione and Xenopus embryos, recruitment of input projection neurons causes faster and more vigorous swimming motor patterns (Arshavsky et al., 1989; Sillar and Roberts, 1993). However, in both cases, the basic temporal structure of the target motor program remains unchanged. In contrast, recruitment of an identified projection neuron that selects a different rhythmic motor program has already been reported in the gastric mill system of the crab (Norris et al., 1994), and activation of a single brain command neuron switches crab scaphognathite movements from forward to reverse ventilatory motor patterns (DiCaprio, 1990). Our present data reveal that different network coordinations can be configured by separate or combined activation of dual input pathways to the same motor circuitry in vitro and that 
the output patterns produced closely resemble two distinct gastric mill motor programs seen during spontaneous rhythmicity in vivo.

\section{The gastric mill system in different species}

The essential features of the Homarus gastric mill CPG are similar to those described previously in other species, such as the spiny lobsters Panulirus and Palinurus (Maynard and Dando, 1974; Mulloney and Selverston, 1974a,b; Hartline and Maynard, 1975) and crabs (Weimann et al., 1991; Katz and Tazaki, 1992). One notable difference, however, is in the number of motoneurons innervating the protractor (gm1) muscle of the gastric medial tooth. Instead of only four GM motoneurons, as in Macrurans and Brachyurans, we find that the gm1 muscle of Homarus is innervated by 10 excitatory motoneurons. This large population of medial tooth power-stroke motoneurons contrasts with the relatively small number of neurons making up the other functional motoneuron subgroups: two for both the power-stroke (LG-MG neurons) and return-stroke (LPG neurons) muscles of the lateral teeth and only one (DG neuron) for the medial tooth returnstroke muscle. The reason for this difference both within the lobster network and between the gastric mill networks of different species is unknown, although our results indicate that the large number of GM neurons in Homarus plays a crucial role in biasing projection neuron input within the gastric mill network itself (see below).

Other interspecies differences involve certain synaptic connections between individual gastric mill network elements. For example, unlike spiny lobsters (Selverston and Moulins, 1987), no evidence was found for a rectifying electrical coupling between GM and LPG. Similarly, an inhibitory synapse from LPG to LG reported in Panulirus interruptus appears to be absent in the European lobster. Despite these phylogenetic differences in synaptic wiring, gastric mill motor output in vitro is remarkably similar in these species, suggesting compensatory changes in cellular properties of individual network neurons and/or that the above connections play a relatively minor role in shaping the final pattern.

\section{Direct versus indirect actions of projection pathways}

Our results again demonstrate the importance of accounting for indirect network effects in understanding precisely how an input pathway operates (Hooper and Moulins, 1990). For example, although the CG interneuron appears to monosynaptically excite the LPG motoneurons of the gastric mill CPG, these neurons are in fact inhibited when the CG neuron fires alone. This apparent paradox derives from the fact that the CG neuron also has a strong inhibitory action on the LPG neuron via a polysynaptic pathway mediated by the GM and LG-MG neurons (Fig. 6). The predominance of the indirect CG to LG-MG neuron excitation is undoubtedly assisted by the relatively large number (10) of GM motoneurons, all of which appear to be directly excited by the interneuron. In a manner equivalent to the convergence of visual information in the vertebrate retina (Sterling et al., 1986), such parallel information transfer via the GM motoneuron population is likely to amplify the signal of the CG interneuron, thereby increasing its influence on the LG-MG neurons. As a consequence, the CG interneuron is able to induce a gastric mill motor output pattern in which the medial tooth protractor GM motoneurons fire in antiphase with the lateral teeth opener LPG motoneurons. It is only when the LG-MG neurons are inhibited by the GI neuron that the CG neuron can induce conjoint GM and LPG neuron activity.

\section{The CG and GI interneurons are components of a sensory feedback loop}

Motor networks can express operational flexibility via a number of different mechanisms (Getting and Dekin, 1985; Marder and Calabrese, 1996), including altering the way afferent information is conveyed to the network. Employment of different input pathways to reconfigure motor circuitry has been reported in several systems in both simple vertebrates and invertebrates. For example, the switch between motor patterns responsible for swimming and struggling in Xenopus tadpole relies on changes in the recruitment of a population of sensory interneurons (Soffe, 1991, 1997), and in the leech different reflex responses to touch derive from sensory information processing in a distributed population of intercalated interneurons (Lockery and Kristan, 1990). The CG and GI neurons are also part of a sensory feedback loop to the gastric mill CPG and are both postsynaptic to the same identified primary mechanoreceptor neuron, the anterior gastric receptor (AGR) (Simmers and Moulins, 1988; Combes et al., 1995, 1999). Thus, the entire sensorimotor system comprises few elements (two CG and GI interneuron pairs intercalated disynaptically between the single AGR neuron and the 16 neuron gastric mill motor network), all of which are accessible for electrophysiological recording and manipulation in vitro. As shown in our accompanying paper (Combes et al., 1999), AGR can activate the GI and CG interneurons in a selective manner, similar to that reproduced by the direct interneuronal stimulation used in the present study. That is, depending on its level of discharge, AGR can activate the interneurons either singly or in combination, and thus via the cellular and synaptic mechanisms identified here induce two different gastric mill network configurations.

\section{REFERENCES}

Arshavsky YI, Orlovsky GN, Panchin YV, Pavlova GA (1989) Control of locomotion in the marine mollusc Clione limacina. VII. Reexamination of the type 12 interneurones. Exp Brain Res 78:398-406.

Blitz DM, Nusbaum MP (1997) Motor pattern selection via inhibition of parallel pathways. J Neurosci 17:4965-4975.

Clemens S, Combes D, Meyrand P, Simmers J (1998) Long term expression of two interacting motor pattern generating networks in the stomatogastric system of freely behaving lobster. J Neurophysiol 79:1396-1408.

Combes D, Simmers J, Nonnotte L, Moulins M (1993) Tetrodotoxinsensitive dendritic spiking and control of axonal firing in a lobster mechanoreceptor neurone. J Physiol (Lond) 460:581-602.

Combes D, Simmers J, Moulins M (1995) Structural and functional characterization of a muscle tendon proprioceptor in lobster. J Comp Neurol 363:221-234.

Combes D, Meyrand P, Simmers J (1999) Dynamic restructuring of a rhythmic motor program by a single mechanoreceptor neuron in lobster. J Neurosci 19:3620-3628.

Delcomyn F (1980) Neural basis of rhythmic behavior in animals. Science 210:492-498.

DiCaprio RA (1990) An interneurone mediating motor programme switching in the ventilatory system of the crab. J Exp Biol 154:517-535.

Getting PA, Dekin MS (1985) Tritonia swimming. A model system for integration within rhythmic motor systems. In: Model neural networks and behavior (Selverston AI, ed), pp 3-20. New York: Plenum.

Harris-Warrick RM, Marder E, Selverston AI, Moulins M (1992) Dynamic biological networks: the stomatogastric nervous system. Cambridge, MA: MIT.

Hartline DK, Maynard DM (1975) Motor patterns in the stomatogastric ganglion of the lobster Panulirus argus. J Exp Biol 62:405-420.

Heinzel HG (1988) Gastric mill activity in the lobster. I. Spontaneous modes of chewing. J Neurophysiol 59:528-550.

Hooper SL, Marder E (1984) Modulation of a central pattern generator by two neuropeptides, proctolin and FMRF amide. Brain Res 305:186-191.

Hooper SL, Moulins M (1990) Cellular and synaptic mechanisms re- 
sponsible for a long-lasting restructuring of the lobster pyloric network. J Neurophysiol 64:1574-1589.

Katz PS, Harris-Warrick RM (1989) Serotonergic/cholinergic muscle receptor cells in the crab stomatogastric nervous system. II. Rapid nicotinic and prolonged modulatory effects on neurons in the stomatogastric ganglion. J Neurophysiol 62:571-581.

Katz PS, Tazaki K (1992) Comparative and evolutionary aspects of the crustacean stomatogastric system. In: Dynamic biological network: the stomatogastric nervous system (Harris-Warrick RM, Marder E, Selverston AI, Moulins M, eds), pp 221-261. Cambridge, MA: MIT.

Katz PS, Eigg MH, Harris-Warrick RM (1989) Serotonergic/cholinergic muscle receptor cells in the crab stomatogastric nervous system. I. Identification and characterization of the gastropyloric receptor cell. J Neurophysiol 62:558-570.

Lockery SR, Kristan Jr WB (1990) Distributed processing of sensory information in the leech. I. Input-output relations of the local bending reflex. J Neurosci 10:1811-1815.

Marder E, Calabrese RL (1996) Principles of rhythmic motor pattern generation. Physiol Rev 76:687-717.

Maynard DM, Dando MR (1974) The structure of the stomatogastric neuromuscular system in Callinectes sapidus, Homarus americanus and Panulirus argus. Phil Trans R Soc Lond B Biol Sci 268:161-220.

Meyrand P, Simmers J, Moulins M (1994) Dynamic construction of a neural network from multiple pattern generators in the lobster stomatogastric nervous system. J Neurosci 14:630-644.

Miller JP, Selverston AI (1979) Rapid killing of single neurons by irradiation of intracellular injected dye. Science 206:702-704.

Morton DW, Chiel HJ (1994) Neural architectures for adaptive behavior. Trends Neurosci 17:413-420.

Mulloney B, Selverston AI (1974a) Organization of the stomatogastric ganglion in the spiny lobster. I. Neurons driving the lateral teeth. J Comp Physiol 91:1-32.

Mulloney B, Selverston AI (1974b) Organization of the stomatogastric ganglion in the spiny lobster. III. Coordination of the two subsets of the gastric system. J Comp Physiol 91:53-78.

Norris BJ, Coleman MJ, Nusbaum MP (1994) Recruitment of a projection neuron determines gastric mill motor pattern selection in the stomatogastric nervous system of the crab, Cancer borealis. J Neurophysiol 72:1451-1463.

Robertson RM, Moulins M (1981) Firing between two spike thresholds: implications for oscillating lobster interneurons. Science 214:941-943.

Selverston AI, Moulins M (1987) The crustacean stomatogastric system. Heidelberg: Springer.

Sillar KT, Roberts A (1993) Control of frequency during swimming in Xenopus embryos: a study on interneuronal recruitment in a spinal rhythm generator. J Physiol (Lond) 472:557-572.

Simmers J, Moulins M (1988) A disynaptic sensorimotor pathway in the lobster stomatogastric system. J Neurophysiol 59:740-756.

Soffe SR (1991) Triggering and gating of motor responses by sensory stimulation: behavioural selection in Xenopus embryos. Proc R Soc Lond B Biol Sci 246:197-203.

Soffe SR (1997) The pattern of sensory discharge can determine the motor response in young Xenopus tadpoles. J Comp Physiol [A] 180:711-715.

Sterling P, Freed M, Smith RG (1986) Microcircuitry and functional architecture of the cat retina. Trends Neurosci 9:186-192.

Weimann JM, Meyrand P, Marder E (1991) Neurons that form multiple pattern generators: identification and multiple activity patterns of gastric/pyloric neurons in the crab stomatogastric system. J Neurophysiol 65:111-122. 